\title{
A communication-assisted protection scheme for direct-current distribution networks
}

\author{
Mehdi Monadi a, b, *, M. Amin Zamani ${ }^{\mathrm{c}}$, Cosmin Koch-Ciobotaru ${ }^{\mathrm{d}}$, Jose Ignacio Candela ${ }^{\mathrm{a}}$, \\ Pedro Rodriguez ${ }^{\text {a, }}$ d \\ a Technical University of Catalonia, Barcelona, Spain \\ b Shahid Chamran University of Ahvaz, Ahvaz, Iran \\ ${ }^{c}$ Quanta Technology, Toronto, Canada \\ d Abengoa Research, Seville, Spain
}

Keywords:

Communication-assisted protection

Distributed generation

Real-time simulation

Smart distribution systems

Voltage source converters

\begin{abstract}
A B S T R A C T
One of the major issues associated with the implementation of direct-current distribution systems is the design of a proper protection scheme. The fault current characteristics in direct-current distribution systems are quite different than those in conventional alternating-current grids. Thus, the performance of conventional protection schemes can adversely be affected, and it is necessary to modify the conventional protection schemes or design new protection methods for direct-current networks. This paper proposes a multi-zone differential protection scheme for direct-current distribution systems embedding distributed generators. The proposed method provides a selective and fast protection through the use of a communication link between two sides of a protected feeder. Moreover, the method provides a differential-based backup for the adjacent relays, which can enhance the protection system reliability. In addition, the method proposed in this paper also utilizes directional over-current elements to provide backup protection if the communication network fails. The effectiveness of the proposed protection scheme is evaluated through comprehensive hardware-in-the-loop simulation studies to obtain more realistic results and to investigate the impact of the communication delay. The results show that the proposed method can provide a selective and fast protection and effectively protect components of direct-current distribution systems against different types of faults.
\end{abstract}

\section{Introduction}

The deployment of RES (Renewable Energy Systems), such as PV (Photovoltaic) systems, wind power systems, fuel cells, and microgas turbines, is increasingly growing in electric distribution networks [1]. Some of these non-conventional distributed energy resources produce DC (Direct Current) power, or include a DC stage, which makes it necessary to utilize an AC/DC stage [2]. This is the reason why most of the RES-based DGs (distributed generators) are interfaced with the host AC (Alternating Current) grid through power-electronic converters consisting of AC/DC (AC to DC conversion) and $\mathrm{DC} / \mathrm{AC}$ ( $\mathrm{DC}$ to $\mathrm{AC}$ conversion) power conversion stages

\footnotetext{
* Corresponding author. Research Center on Renewable Electrical Energy Systems (SEER Center), GAIA building, 22, Rambla Sant Nebridi, 08222, Campus Terrassa, Barcelona, Spain. Tel.: +34 661153290; fax: +34 937398225.

E-mail address: meh_monadi@yahoo.com (M. Monadi).
}

[3]. Accordingly, the use of DC systems can prevent unnecessary costs and losses associated with power conversion stages in $\mathrm{AC}$ grids.

DC networks have some other advantages over AC networks. For instance, power losses in DC systems are significantly lower than those in AC systems, thus improving the overall network efficiency [4]. Likewise, in DC networks, it is possible to control the power flow more easily and quickly than AC systems [5]. In addition, DC systems can be used as an interface to connect asynchronous AC grids [6]. Further, energy storage systems can be interfaced with DC systems through the simpler and more efficient converters [7].

Due to the aforementioned reasons, DC grids will be an inevitable and important part of the future distribution systems [8]. Moreover, MVDC (medium-voltage DC) distribution systems are introduced as an alternative for AC systems in future commercial and industrial grids [9]. However, despite their perceived advantages, DC distribution systems also introduce new challenges to the operational principle of power systems. One of the main concerns 


\begin{tabular}{|llll|}
\hline Nomenclature & $\begin{array}{l}\text { MVDC } \\
\text { O/C }\end{array}$ & $\begin{array}{l}\text { medium-voltage DC } \\
\text { over current }\end{array}$ \\
AC & alternating current & OPAL-RT real-time simulator from OPAL-RT Technology \\
ACCB & AC circuit breaker & PG & pole to ground \\
AC/DC & AC to DC conversion & PP & pole to pole \\
ANN & artificial neural network & PV & Photovoltaic \\
CB & circuit breaker & RES & renewable energy system \\
CT & current transducer & SSCB & solid-state circuit breaker \\
DC & direct current & TD & time delay for forward faults \\
DC/AC & DC to AC conversion & TD & time delay for reverse faults \\
DCCB & DC circuit breaker & TM & time margin of directional O/C backup units \\
DG & distributed generator & TMD & time margin of the differential-based backup zone \\
HIL & hardware in the loop & VSC & voltage source converter \\
Inst. & Instantaneously & & \\
\hline
\end{tabular}

associated with DC systems is the implementation of an effective protection scheme [10].

Most recent DC networks use VSCs (voltage source converters) to connect to AC grids and RESs [11]. The characteristics of fault currents in VSC-based DC networks are quite different than those in AC grids; therefore, the performance of conventional protection schemes can adversely be affected. On the other hand, considering the low fault tolerance of VSCs, DC systems need to implement relatively faster protection methods and interrupting devices [12]. This, in turn, imposes strict constraints on the implementation of time-consuming methods of fault detection and fault location for DC systems.

Although different types of fault detection techniques have been proposed for DC systems, the protection methods of DC distribution systems with DGs are still in the early stage of development. Conventional distribution systems are passive systems mostly with radial topology. These networks are typically protected by $\mathrm{O} / \mathrm{C}$ (overcurrent) relays, based on the assumption that the power flow is unidirectional. However, the integration of DGs is forcing the passive distribution systems to change towards active networks [13]. Also, it has been shown in other publications, such as [14], that the integration of DGs changes the radial topology of distribution feeders. Hence, the connection of a DG to a distribution feeder may cause bi-directional power flow and, hence, a change of the fault current direction on that feeder [15]. Consequently, the coordination of conventional $\mathrm{O} / \mathrm{C}$ relays can be disrupted and the protection may fails. In addition, as it has been explained in Ref. [15], due to the high rising rate of fault currents in VSC-based DC networks, it is very difficult to discriminate between faults that occur inside the main protection zone of a relay and outside faults. This is another factor that increase the complexity of $\mathrm{O} / \mathrm{C}$ relays coordination in $\mathrm{DC}$ systems.

To improve the performance of $\mathrm{O} / \mathrm{C}$ protection in DC systems, Reference [16] has suggested using the magnitude of the DC-link voltage along with the fault current. A similar work has been presented in Ref. [17] where a hybrid relay, consisting of overcurrent and under-voltage elements, has been proposed for DC systems. These hybrid relays send the trip command when both the overcurrent and the voltage-based element pick up; therefore, it can reduce the possibility of mal-operation of the O/C-based relay. These methods, however, rely on the measurement of the fault current magnitude; thus, they are still vulnerable to the high rising rate of the DC fault current. In order to prevent the negative impacts of the rapid increment of DC fault currents on the operation of $\mathrm{O} / \mathrm{C}$ relays, it has been suggested in Ref. [18] to analyse the slope of the DC fault current instead of its magnitude. Other authors have proposed to investigate the fault current derivative in order to estimate the fault location [19]. Furthermore, to discriminate between faults inside and outside the main protection zone of a relay, the derivation of both voltage and current have been used in Ref. [20]. Compared to the conventional O/C relays, these methods can provide a relatively improved performance in DC networks. However, these methods rely on O/C-based elements, which will increase the possibility of the protection system failure when DGs are integrated into the network and bi-directional currents flow through the protected feeder.

Since it is difficult to coordinate $\mathrm{O} / \mathrm{C}$ relays in DC networks, Reference [12] has proposed a distance protection scheme for these networks. In this method, the distance from the fault point to the location of the relay is estimated. If the fault is recognized inside the protection zone of the relay, the relay will operate instantaneously; otherwise, it will operate as a backup for the relay of the faulty zone. This operation can provide a selective protection and prevent the operation of relays for faults that happened outside their protection zone. However, the main challenge associated with this technique is the impact of the fault impedance on the accuracy of the method, particularly for short distribution lines. Another method for estimating the fault location has been presented in Ref. [21], where the faults are located by estimating the equivalent impedance from the fault point to the location of the relay by using an AIE (active impedance estimation) method. In this method, a spread frequency current is injected to the DC feeder, and the resultant voltage is recorded. The measured voltage and current are then used to calculate the equivalent impedance. The relay will operate if the estimated impedance is less than the impedance of the protected feeder. However, the choice of a proper frequency for the injected current and the impact of the fault impedance are the main issues of this method.

ANN (artificial neural network)-based methods have been used for fault diagnosis in various filed of power systems [22]. These methods have also been suggested for the protection of DC systems. For example, an ANN-based protection method has been proposed in Ref. [23] where the sampled value of the measured DC voltage and current are directly fed to an ANN-based fault locator. The fault locator, then, calculates the exact location of the fault and sends the trip command if the fault has happened inside the protection zone. However, the direct use of voltage and current samples not only increases the required time for the learning process, but also increases the possibility of protection mal-operation due to the noise and incorrect sampling. To enhance the accuracy of ANN-based method, Reference [24] has suggested analysing the input data before feeding the ANN. In this method, the wavelet transform has 
been used to analyse the measured voltage and current and extract a feature vector from the sampled quantities. Besides the ANNbased methods, other signal-processing based methods have also been presented for protection of DC systems. In Ref. [25], faults in DC networks are detected by analysing the wavelet-transform coefficients of voltage and current along with the magnitude and derivative of the DC bus voltage. The aforementioned coefficients are continuously calculated and if their values exceed a predetermined threshold, the relay detect the fault inside the main protection zone and sends the trip command to the associated $\mathrm{CB}$ (Circuit Breaker). In addition to wavelet-based methods, the method presented in Ref. [26] shows that Fourier-transform can also be utilized to analyse the measured current and detect faulty DC feeders. In all the aforementioned signal-processing based methods, specific features of the voltage and current waveforms are extracted to detect and/or locate network faults. Although, these methods have shown promising results, the extracted features are not necessarily the same for all DC systems; thus, they cannot provide a generic approach for the protection of DC networks. Moreover, the need to a relatively faster protection algorithm in DC systems limits the application of these method in DC networks. On the other hand, the travelling-waves-based protection methods have been suggested in Ref. [27] for DC lines. When a fault happens in a DC line, the travelling waves are propagated from the fault location to both ends of the faulty line. Synchronized measurements are able to determine the accurate difference between the arrival times of the travelling waves to each end of the line. According to this time difference and considering the velocity of the travelling waves, the exact location of the fault can be calculated. However, this method is more applicable for long distance lines and, due to the small length of distribution feeders, it is very difficult to obtain the exact time difference and locate the exact location of the fault.

Analytical methods based on the investigation of voltage and current are effective methods for fault detection in power networks [28]. Accordingly, the faulty DC feeder can be detected by means of a mathematical analysis of the current flowing through the fault path [29]. The fault path of a typical VSC-based network is shown in Fig. 1. According to this figure, it is suggested in Ref. [29] to connect a probe power unit to each end of the DC feeder. The probe includes a capacitor which is charged by an internal source. After the fault occurrence and once the DCCBs (DC circuit breakers) are opened in both sides of a line, the capacitor of the power probe is connected to the line. If the fault occurs inside the protected line, the capacitor is discharged through the fault path. Then, the exact location of the fault is determined by analysing the discharge current of this capacitor. However, in this method, CBs of all the healthy lines are opened and reclosed when the faulty line was recognized and isolated. Therefore, the use of this method for the medium voltage networks may cause power quality issues.

Over these years, with the advent of smart grids, more accurate and effective methods have been presented to improve the operation of power systems [30]. Therefore, it is predicted that the future power systems are mostly designed based on the operational features of smart grids [31]. By means of using the communication infrastructures of smart grids, communication-assisted methods has been implemented in AC distribution systems embedding DGs [32]; these methods can also provide effective protection for DC systems. Differential protection, as an communication-assisted method, has been proposed as an effective and fast method for the protection of DC distribution systems [33]. In fact, since the fault current level, the rate-of-change of the current, status of DGs, and fault resistance have relatively low impact on the performance of differential protection, they are one of the best options for the protection of DC systems embedding DGs [15]. Therefore, in Ref. [34] conventional differential relays have been used to provide a selective protection for multi-terminal DC systems. Also, a more accurate differential-based relay equipped with operating and restraining signals has been presented in Ref. [35]. The operating and restraining signals are generated based on the energy indices of each line to ensure accurate decision of the relay and to prevent the operation of the relay for the external faults. In this relay, when a fault occurs outside the main protection zone, the restraining signals will be much higher than operating signal and hence tends to restrain the operation of the relay. However, as opposed to non-unit protection schemes such as $\mathrm{O} / \mathrm{C}$ and distance protection, conventional differential schemes cannot provide backup protection for adjacent protection zones. Moreover, since the effectiveness of differential protection heavily relies on the existence of a reliable communication medium, any communication failure can substantially affect the performance of the protection. Therefore, it is important to apply some modifications to the conventional differential protection in order to deal with these issues.

This paper proposes a multi-zone differential-based protection method for MVDC distribution systems. The proposed communication-assisted method employs a simple and solid algorithm that does not require time-consuming processes, while providing second and third differential zones that operate as a backup for the main zone. More importantly, the proposed method is equipped with directional $\mathrm{O} / \mathrm{C}$ relays which provide a selective

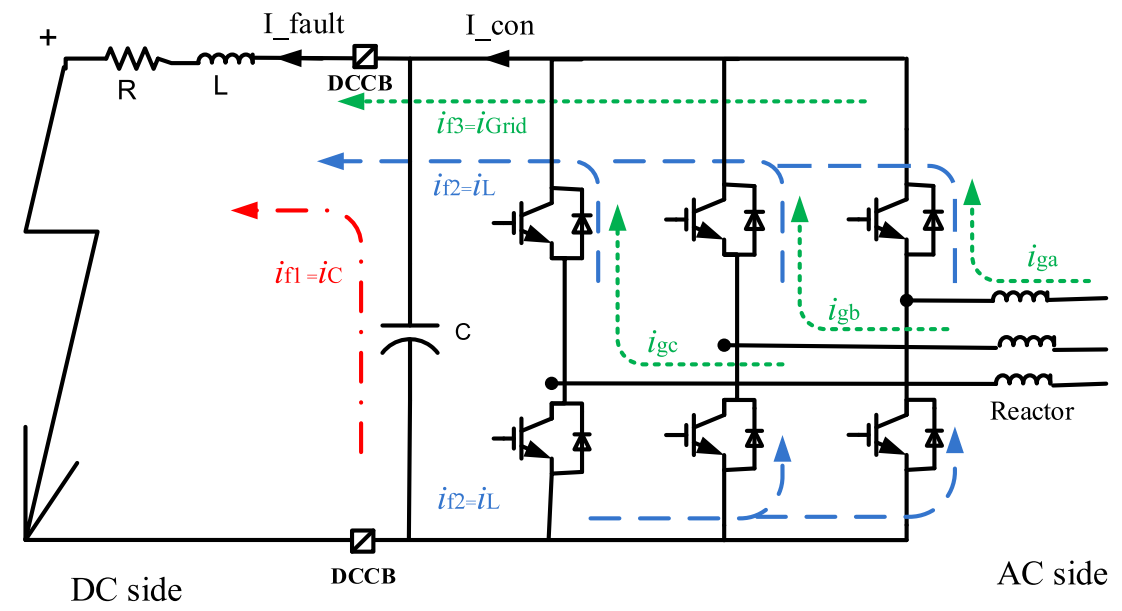

Fig. 1. Equivalent circuit of a PP fault in a VSC-based DC feeder [15]. 
backup protection if the communication system fails. The contributions of this paper can be summarized as the following:

1) The proposed multi-zone differential relay provides backup differential protections, besides the conventional differential protection, to improve the reliability of the overall protection system.

2) The proposed method also provides a directional overcurrentbased backup protection to manage communication failures.

3 ) The effectiveness of the proposed method is independent of the network operational mode, fault current levels, and status of DGs. Therefore, it is a suitable performance for distribution systems with a high penetration of DGs.

4) The efficacy of the proposed technique is demonstrated through real-time HIL (hardware in the loop) testing conducted in a realtime simulator (OPAL-RT) to incorporate the impact of communication delays.

The rest of the paper is organized as follows. In Section 2, the specification of DC fault currents are described, and the fault interruption methods are introduced. The components of the main and backup units are introduced in Section 3, while the proposed protection strategy is explained in Section 4. Section 5 describes the HIL simulation method which is used for the evaluation of the proposed method. Results of the implementation of the proposed method are finally shown and discussed in Section 5.

\section{Fault current in VSC-based DC networks}

\subsection{Characteristics}

In order to design an effective protection scheme for DC distribution systems, it is imperative to analyse the characteristics of DC fault currents. Fig. 1 shows a simplified schematic diagram of a typical PP (pole-to-pole) fault in a VSC-based DC feeder. In this figure, $\mathrm{R}$ and $\mathrm{L}$ represent the resistance and inductance of the line located between the main DC bus (DC-link capacitor) and the fault location, respectively. Subsequent to the fault occurrence in the DC grid, the DC-link capacitor is discharged due to the voltage drop of the main DC bus. Once the DC-link capacitor is discharged, the energy stored in the cable inductance is discharged through the freewheeling diodes of the VSC. On the other hand, the control of the converter turns off the main switches of the VSC to protect them against the over-current condition caused by faults; this causes the VSC to operate as an uncontrolled full-bridge rectifier and continue feeding the fault from the AC side through the freewheeling diodes [15].

Based on the above discussion, the DC fault current includes three different components, namely, $i$ ) the DC-link capacitor discharging current $\left.\left(i_{C}\right), i i\right)$ the cable inductance discharging current $\left(i_{L}\right)$, and $\left.i i i\right)$ the AC grid current $\left(i_{\text {Grid }}\right)$. The components of a typical DC fault current are shown in Fig. 2. During the capacitor discharging period $\left(t_{0} \leq t \leq t_{c}\right)$, there is no fault current contribution from the converter side. However, for $t>t_{c}$, the inductor discharging current and the AC grid current flow through the converter freewheeling diodes (see Fig. 2(b)); this may damage the freewheeling diodes of the VSC [29]. Therefore, the protection of DC distribution systems should operate during the capacitor discharging component $\left(\mathrm{t}_{0} \leq \mathrm{t} \leq \mathrm{t}_{\mathrm{c}}\right.$ ) to prevent any damage to the converter [20]. This is based on the assumption that, subsequent to the fault, the converter remains in-service in order to give a chance to the protection system to isolate the fault. This also ensures that the smallest possible area of the system is de-energized in response to the fault.

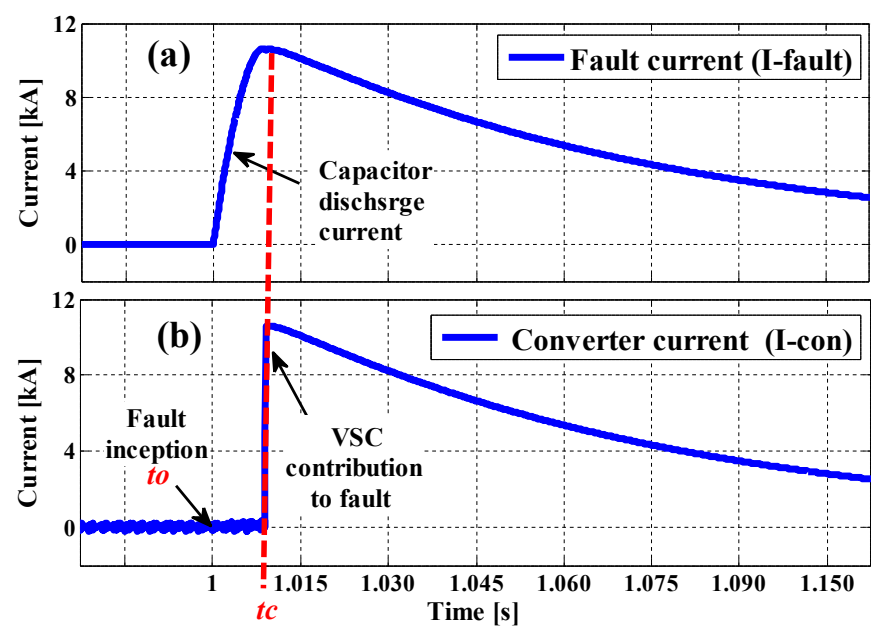

Fig. 2. Waveforms of a typical DC fault current. Fig. 1.

The critical time for the operation of the DC protection systems, that is, $\Delta t_{c}=t_{c}-t_{0}$, depends on several factors such as fault type, fault location, and fault resistance. For a typical PP fault, the critical time can be calculated as [36]:

$\Delta t_{c}=\left(\pi-\arctan \left(\left(V_{0} C \omega_{0} \sin (\beta) /\left(V_{0} C \omega_{0} \cos \beta-I_{0}\right) / \omega\right)\right.\right.$

where $\omega=\sqrt{\frac{4 L-R^{2} C}{4 L^{2} C}} ; \beta=\arctan \left(\frac{2 \omega L}{R}\right) ; I_{0}=i_{\text {Cable }}(0) ; V_{0}=v_{c}(0)$; $\omega_{0}=\frac{1}{\sqrt{L C}}$.

It should be pointed out that, for high impedance faults, the critical time increases; this also may happen in the case of PG (poleto-ground) faults. In addition, although the fault current characteristics of a two-level converter have been studied in this study, the analyse is the same for multi-level converters and half-bridge modular multilevel converters [35].

\subsection{Interruption methods}

This section gives a brief overview of different fault interruption methods in DC systems. It is well known that conventional ACCBs (AC circuit breakers) interrupt the fault current during a zero crossing of the current waveform. Since DC fault currents do not cross zero, ACCBs are not suitable for interrupting these fault currents. The use of $\mathrm{AC}$-side $\mathrm{CBs}$ along with the converter blocking scheme and DC isolating switches have been proposed for fault interruption in multi-terminal DC systems [37]. The main disadvantage of this method is that ACCBs may not operate fast enough to prevent damages to freewheeling diodes of the converter. Moreover, ACCBs are not capable of breaking the DC-link capacitor discharging current, $i_{c}$. It should also be noted that the aforementioned methods isolate the faulty parts of the network based on a "cut-and-try" process, which can significantly affect the reliability of the DC and AC-side network. Therefore, this paper assumes that all feeders are equipped with DCCBs (DC circuit breakers), as suggested in Ref. [38]. The solid-state DCCBs are capable of fault clearance in sub-millisecond [39]. It is, however, acknowledged that the economic aspects related to the widespread use of DCCBs are still under investigation.

\section{Components of the proposed protection method}

It was discussed in Section 1 that although differential protection is a well proven technique for fast protection of DC distribution lines, it cannot operate as a backup for adjacent protection zones. 


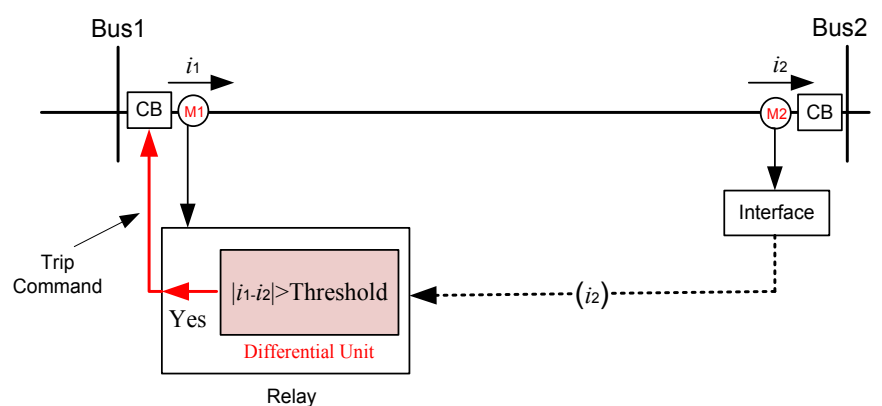

Fig. 3. Differential protection scheme for a distribution line.

Whereas, to provide a reliable protection, each protected zone should be protected by at least one backup protection [40]. The lack of backup protection and the need for a reliable communication medium challenge the application of differential protection for MVDC distribution feeders. This paper proposes a modified differential-based protection scheme which is capable of $i$ ) providing backup protection, ii) managing the communication network failure, and iii) operating correctly in the existence of DGs with various operational modes. In the following subsections, the main elements of the proposed method are introduced.

\subsection{Main differential protection}

The proposed method is mainly based on the differential algorithm in which the difference between the currents in both sides of a protected feeder/zone is continuously measured. If the absolute value of this differential current is found to be greater than a threshold, a trip signal is sent to the corresponding DCCB in each ends of the line. For the relay shown in Fig. 3, the absolute value of differential current is computed as:

$i_{\text {diff }}[k]=\left|i_{1}[k]-i_{2}[k]\right|$

where $i_{1}$ and $i_{2}$ denote the measured current at both ends of the protected line, and $k$ is the sample number. Equation (2) shows that bi-directional power flows caused by DGs cannot impact the performance of the differential scheme.

\subsection{Main and backup protection zones}

To ensure the protection system reliability, the proposed protection strategy provides main and backup protection zones. These protection zones are divided into two groups as follows.

\subsubsection{Differential-based protection zones}

In the proposed method, each relay provides main and backup differential-based protection zones. For example, for relay R3 shown in Fig. 4, these protection zones are:

1 Zone 1: It is the main protection zone covering the main line whose should be protected by the relay (i.e., Line23 in this example). Thus, the relay operates instantaneously for any fault taking place in this line.

2 Zone 2: It is a backup protection zone for the downstream line/ busbar of the relay (i.e., Line34 and Bus3 in this example). Therefore, for any fault in this line/bus, the relay operates after a time delay, named as TMD (time margin of the differentialbased backup zone), if the main protection of the line/bus fails to operate.

In other words, the proposed method not only provides a main differential zone (i.e., Zone 1), but also offers a differential backup zone for the neighbouring lines/busbars (i.e., Zone 2). Consequently, adding a differential backup for the neighbouring zones will enhance the reliability of the protection system. For example, let us consider fault F1 in Fig. 4. This fault can be detect by both relays R5 (Zone 1) and R3 (Zone 2). If the relay R5 fails to operate and isolate the fault within a predetermined time (i.e., TMD), relay R3 will operate in its backup zone to clear the fault.

On the other hand, to provide a selective protection without any interference between backup zones, it is necessary to ensure that the Zone 2 of the backup relay does not operate before the Zone 1 of the downstream relay. Therefore, TMD is the key factor in the coordinated operation of the main and backup relays. As explained in Section 2.1, the fault current should be interrupted during the first stage of the fault current. Accordingly, the main and backup zones should operate during the critical time of the VSC station (i.e., $\Delta t_{c}$ ). On the other hand, both Zone 1 and Zone 2 requires communication links; hence, they detect faults after $t_{\text {det }}$ which is heavily dependent on communication delay. Thus, as shown in Fig. 5, once a fault is detected, both the main and backup differential protection should operate within $\mathrm{t}_{\mathrm{mb}}$ time frame that can be calculated as:

$t_{m b} \leq \Delta t_{c}-t_{d e t}$

where $\Delta t_{c}$ is the critical time of the VSC station, and $t_{\text {det }}$ is the fault detection time of the relays (including the communication delay and processing time). Since the $t_{\mathrm{mb}}$ is the maximum time frame that both main and backup zones should operate, the maximum value of TMD can be calculated as:

$$
T M D_{\max }=\mathrm{t}_{\mathrm{mb}} / 2
$$

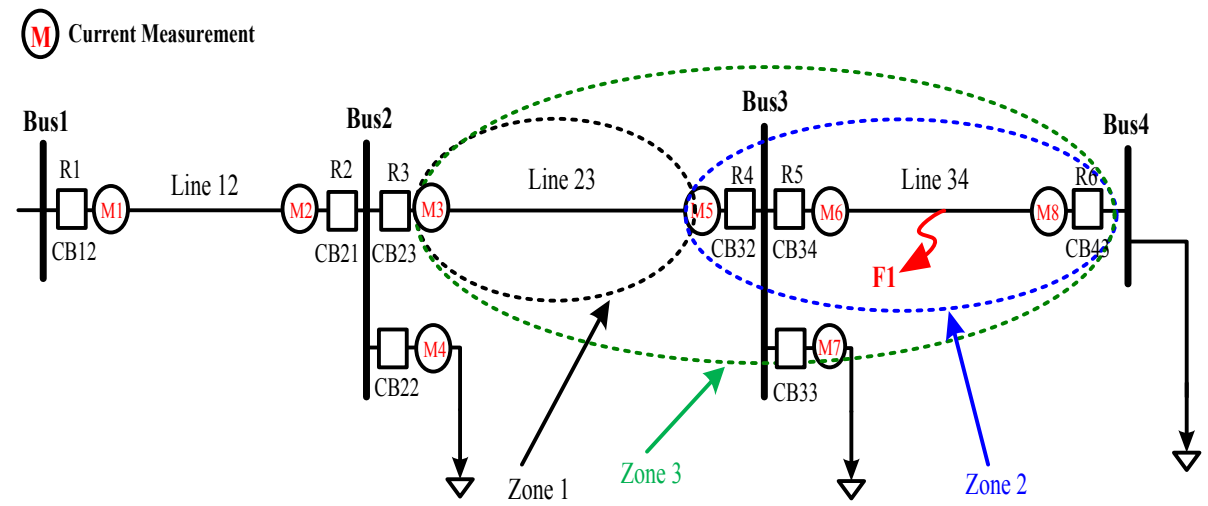

Fig. 4. Proposed differential-based protection zones (for Relay R3). 


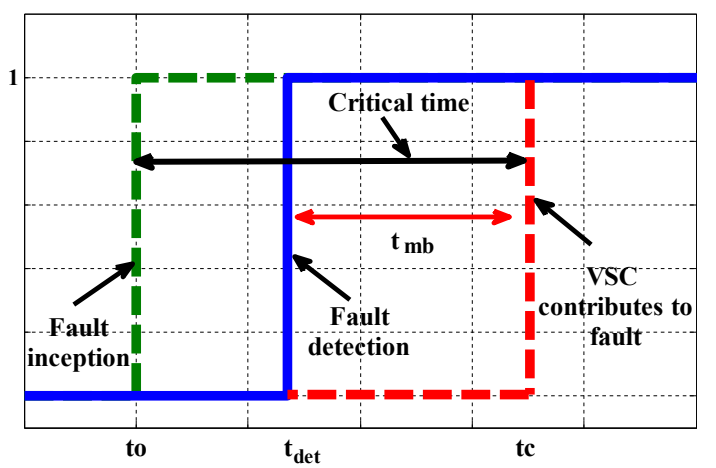

Fig. 5. Typical time frames for fault detection and sending the trip command.

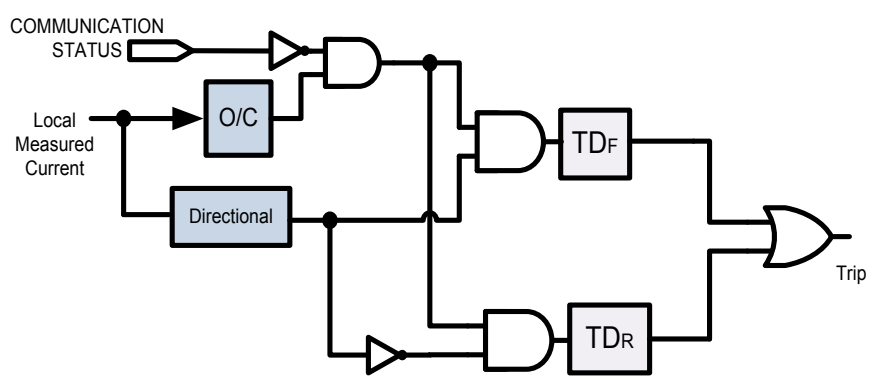

Fig. 6. Symbolic logic circuit for the backup protection.

On the other hand, the minimum value of TMD should be larger than the operating time of DCCBs and the delay associated with CTs (current transducer). Therefore, to provide a selective protection, the time margin between the main and backup zones should be selected as:

$\mathrm{t}_{\mathrm{Br}}<\mathrm{TMD} \leq \mathrm{t}_{\mathrm{mb}} / 2$ where $t_{B r}$ is the summation of the operating time of DCCBs and the delay associated with current transducer and its $A / D$ converters. Equation (5) implies that if in a specific network, $t_{\mathrm{mb}} / 2$ is less than the operating times of the SSCBs (solid-state circuit breakers), there will be a high possibility of mis-coordination between the consecutive relays.

It is also notable that, the proposed method also offers the possibility of a third zone consisting of Zone 1 and Zone 2. Zone 3 can provide a backup protection for the main protection zone (i.e., Line23 in this example). Therefore, if the main protection of Line23 (Zone 1 of R3) fails to operate, e.g., due to a deficiency in M5, Zone 3 can provide backup for Zone 1 .

\subsubsection{O/C directional-based backup protection zones}

Since the differential protection is vulnerable to communication failures, the proposed method employs $\mathrm{O} / \mathrm{C}$ directional relays to back the differential protection up if the communication network fails. The $\mathrm{O} / \mathrm{C}$ directional relays can be of either inverse-time type or definite-time type. Although inverse-time $\mathrm{O} / \mathrm{C}$ relays provide a lower tripping time in large systems, their coordination in DC systems is challenging due to the high rising rate of DC fault currents [41]. The use of backup $\mathrm{O} / \mathrm{C}$ directional protection has been proposed in Ref. [42]. However, this type of coordination may results in a relatively longer operation time in large distribution systems. Therefore, since a fast protection scheme is required for DC networks (to guarantee the safety of the converters), this paper proposes to employ the backup $\mathrm{O} / \mathrm{C}$ directional element for a selective number of relays within the DC network. Thus, in order to minimize the fault clearance time in a large DC network, each feeder of the network is divided into several protection zones. The relay of each zone is then equipped with a directional definite-time $\mathrm{O} / \mathrm{C}$ element. In addition, each protection zone will need to have a radial structure for the proper operation of the backup protection. Fig. 6 shows the logic circuit of the backup protection scheme where the $\mathrm{O} / \mathrm{C}$ directional element have two operating times for forward and reverse faults, i.e., $T D_{F}$ and $T D_{R}$, respectively. As Fig. 6 indicates, if the communication network fails while a forward

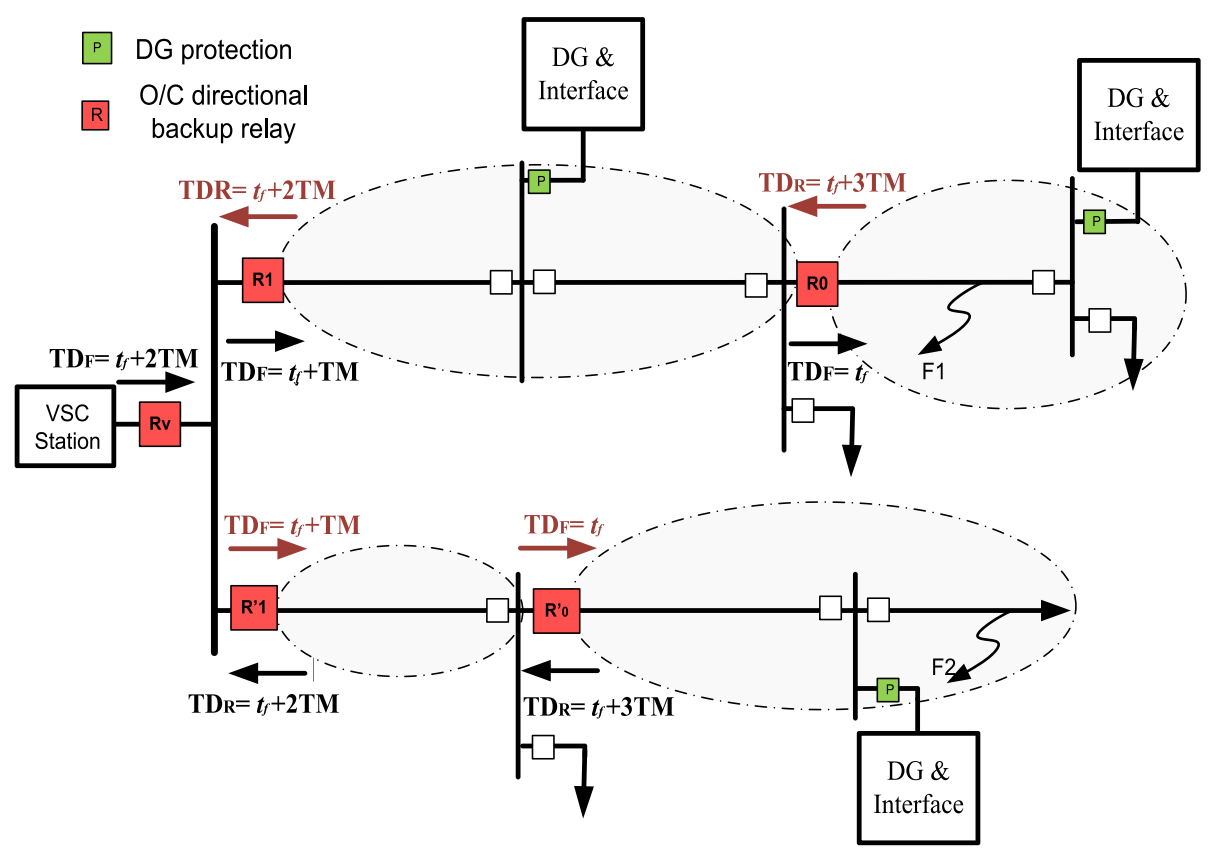

Fig. 7. Coordination of $\mathrm{O} / \mathrm{C}$ directional relays for the backup protection scheme. 
fault is detected, a trip signal is issued after the pre-determined time delay $\left(T D_{F}\right)$.

Fig. 7 shows the coordination of $\mathrm{O} / \mathrm{C}$ definite-time relays for the backup protection scheme in a DC distribution system with two feeders. In this figure, TM (time margin of directional $\mathrm{O} / \mathrm{C}$ backup units) denotes a suitable time margin that enables coordination of relays in forward and reverse directions. It is noted that since SSCBs are used, TM is in the range of several milliseconds (see Section 5; case study 4 ).

The grading times for forward and reverse directions are chosen in a way that the smallest part of the network is de-energized subsequent to a fault. In the network of Fig. 7 each feeder has divided to 2 zones; therefore, to provide a selective protection, the operating time of the most upstream relay (i.e., $R_{V}$ ) is set on:

$T D_{F}\left(\mathrm{R}_{\mathrm{V}}\right)=\mathrm{t}_{\mathrm{f}}+2 \mathrm{TM}$

where $t_{f}$ is the time delay of the operation of the most downstream relay of each feeder. In general case, if assume that the maximum zone number of each feeder is denoted by $n$, the operating time of $\mathrm{R}_{\mathrm{V}}$ can be expressed by:

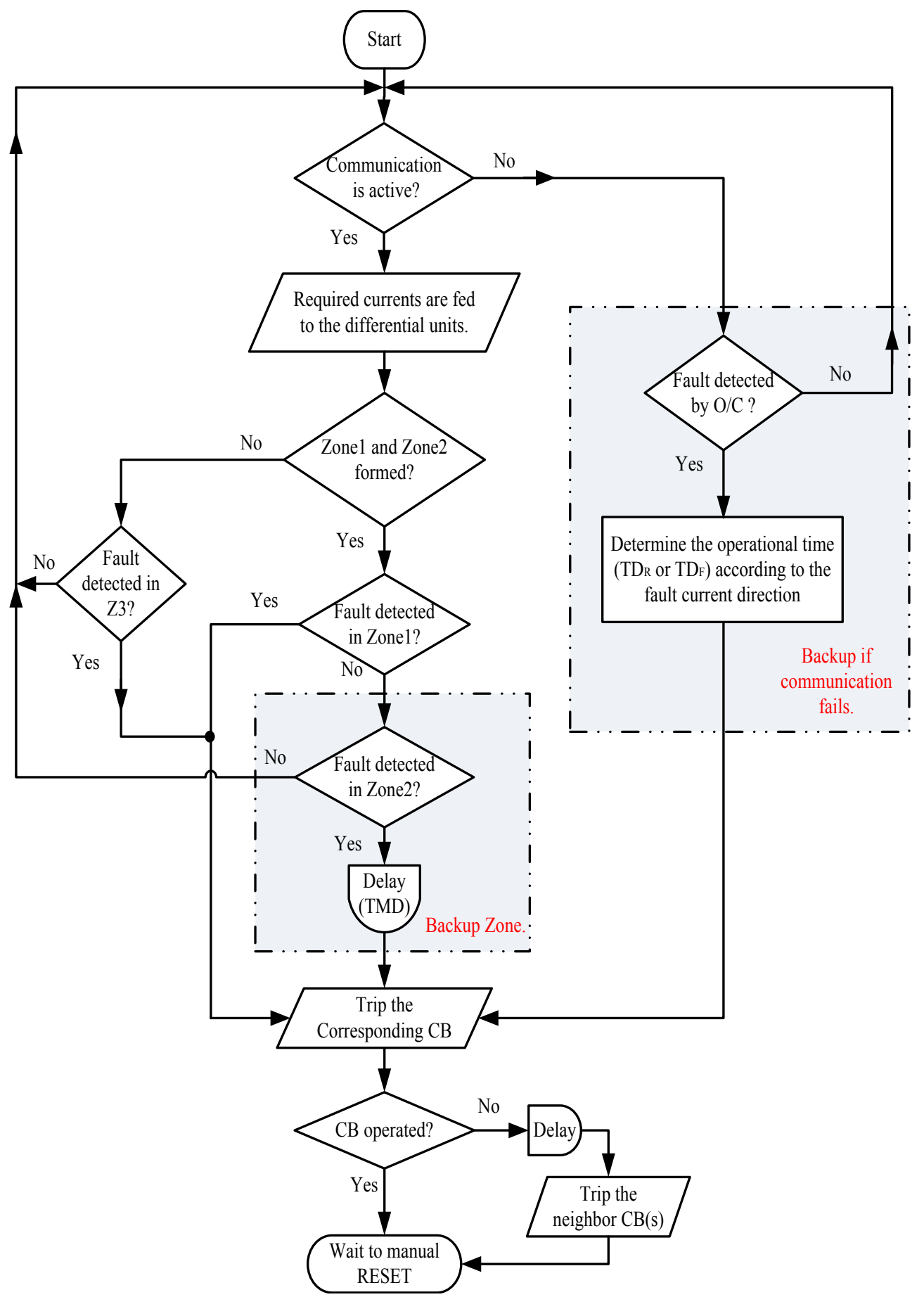

Fig. 8. Flowchart of the proposed protection strategy. 


$$
T D_{F}\left(\mathrm{R}_{\mathrm{V}}\right)=\mathrm{t}_{\mathrm{f}}+\mathrm{nTM}
$$

On the other hand, to protect the VSC station, the maximum operating time of the most upstream relay, i.e., $\mathrm{R}_{\mathrm{V}}$ in Fig. 7, and its corresponding CB should be less than the critical time of the VSC station; that is:

$\Delta \mathrm{t}_{\mathrm{c}}>T D_{\mathrm{F}}\left(\mathrm{R}_{\mathrm{V}}\right)+\mathrm{t}_{\mathrm{Br}}$

Substituting (7) in (8) results in:

$\Delta \mathrm{t}_{\mathrm{c}}>\mathrm{t}_{\mathrm{f}}+\mathrm{nTM}+\mathrm{t}_{\mathrm{Br}}$

Equation (9) indicates that the maximum number of backup zones for each feeder can be calculated as:

$\mathrm{n}<\frac{\Delta \mathrm{t}_{\mathrm{c}}-\mathrm{t}_{\mathrm{Br}}-\mathrm{t}_{\mathrm{f}}}{\mathrm{TM}}$

If no time delay is assumed for the most downstream relays (i.e., $t_{f}=0$ for $R_{0}$ and $R_{0}^{\prime}$ ), then (10) can further be simplified as:

$\mathrm{n}<\frac{\Delta \mathrm{t}_{\mathrm{c}}-\mathrm{t}_{\mathrm{Br}}}{\mathrm{TM}}$

Based on (11), the number of directional $\mathrm{O} / \mathrm{C}$ backup zones is limited by $\Delta \mathrm{t}_{\mathrm{c}}$ and TM. The TM is also determined by the operating time of DCCBs, fault detection time, and the delay associated with CT (current transducer) sensors. Case Study 4 of Section 5 provides more details about the operating times of directional $\mathrm{O} / \mathrm{C}$ units proposed for the backup protection.

\section{Proposed protection strategy}

The flowchart of Fig. 8 provides a graphical illustration of the proposed protection strategy. The current measurement units, i.e., M1 through M8 in Fig. 4, transmit their measured values to the corresponding relays via the communication network. Then, the relay algorithm calculates the differential currents of the protection zones (i.e., Zone 1, Zone 2, and Zone 3). If a fault strikes the line, the differential current of Zone 1 exceeds its respective threshold, and the relay will immediately send trip signal to the associated DCCB. Moreover, Fig. 8 illustrates that if a relay fails to properly detect a fault, the backup differential zone of the upstream relay will operate after a time delay (i.e., TMD). As explained in Section 3.2.1, the TMD is defined in a way that the coordination between the main and backup protection is preserved. Finally, Zone 3 provides a

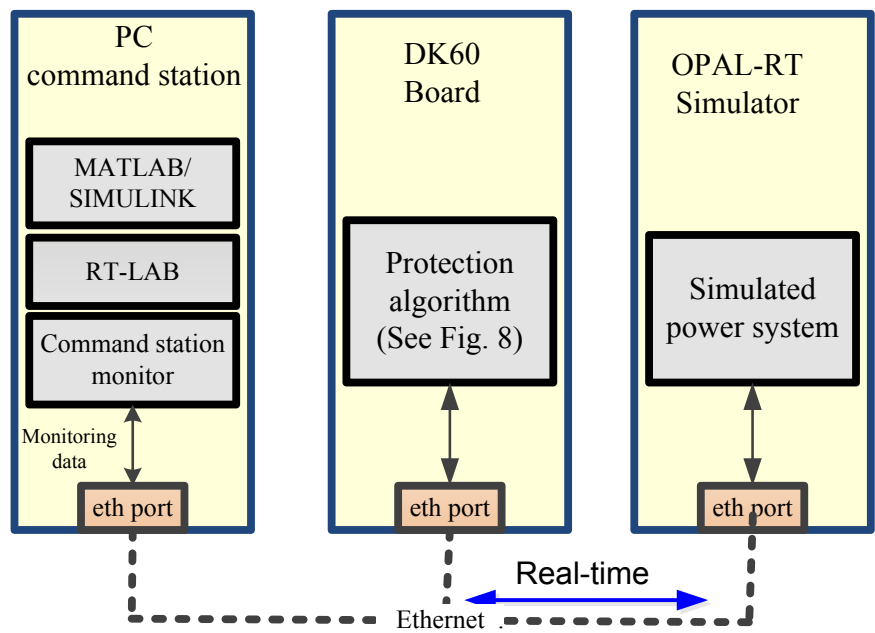

Fig. 9. The schematic diagram of the HIL setup. backup for Zone 1 of the same relay if the measurement device at the remote end of the line fails. It is noted that, considering typical data propagation delays and limited geographical span of a DC distribution system, all relays receive current data and detect faults almost at the same time. The proposed strategy also considered the possibility of $\mathrm{CB}$ failure, that is, if the $\mathrm{CB}$ commanded by the main protection fails to operate, a breaker failure signal is sent to the adjacent $\mathrm{CB}(\mathrm{s})$ to de-energize the smallest possible area of the system.

The flowchart of Fig. 8 also demonstrates that the relay switches to the $\mathrm{O} / \mathrm{C}$ directional-based backup protection if the communication network fails. In this case, the relay will also send an alarm signal to the control centre to report the communication failure. As mentioned in Section 3.2.2, only a selected number of relays are equipped with this backup protection scheme. It should be noted that, according to the specifications of the industrial protocols, the protective devices, are equipped with the communication failure detection capability and switch to the backup protection without requirement to any signal from the external supervisory systems [43].

\section{Real time simulation results}

\subsection{Test setup}

To evaluate the effectiveness of the proposed protection strategy, HIL approach has been used. Fig. 9 shows the schematic diagram of the HIL test-bed setup. The components of this setup are shown in Fig. 10, which include: 1) a real-time digital simulator (OPAL-RT) that simulates the behaviour of the study network components during various operational conditions; 2) a PC that is used as a command station (programming host) to run the Matlab/ Simulink models executed on the OPAL-RT; 3 ) a development board (DK60 from Beck company) which has been used to implement the proposed protection algorithm; and 4) a router that is used to

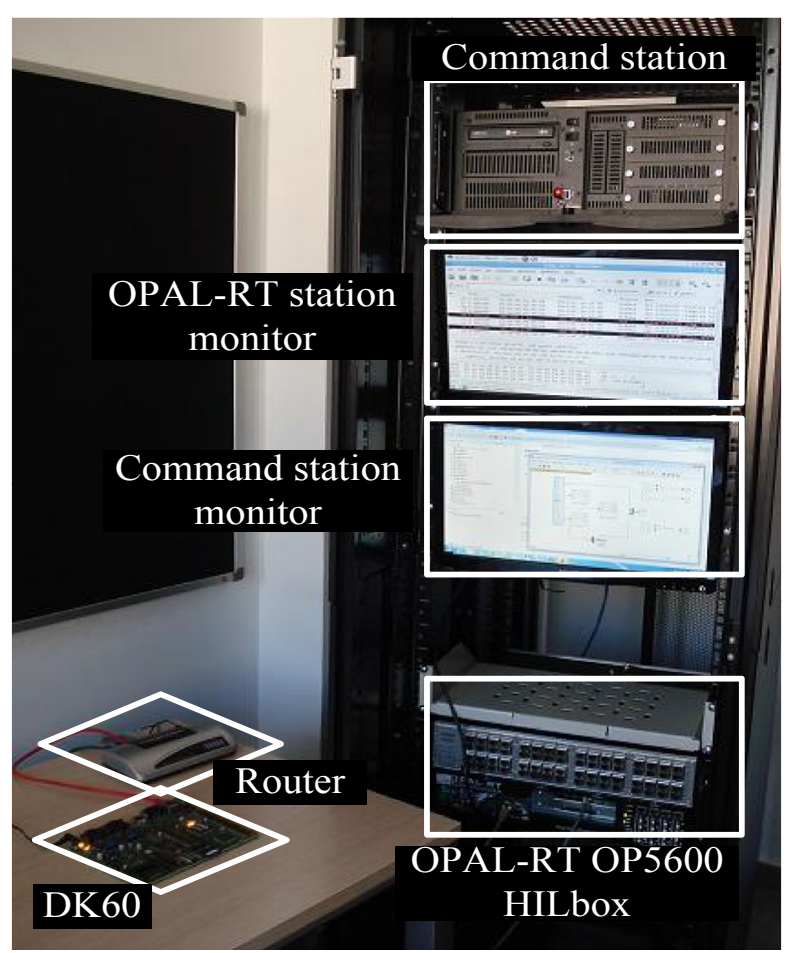

Fig. 10. Components of the HIL setup. 
connect all the setup devices in the same sub-network. In this test setup, the board operates as the protection brain and communicates with current measurements units simulated in the OPAL-RT, via Ethernet medium. Therefore, the HIL test setup considers the communication delays consisting of $i$ ) preparation of data (packaging) in the sending end and ii) opening of the data package in the receiving node. Since the delay associated with data propagation between two nodes is relatively small (less than $0.1 \mathrm{~ms}$ for most distribution feeders [44]), it is ignored in this study.

\subsection{Study system}

The effectiveness of the proposed method is demonstrated in the context of the distribution network of Fig. 11. The basic configuration and parameters of this network is extracted from the benchmark proposed in Ref. [45]; however, some modifications have been done to enable its operation as a DC distribution system with DGs. Hereafter, the network of Fig. 11 is referred to as the "study system".

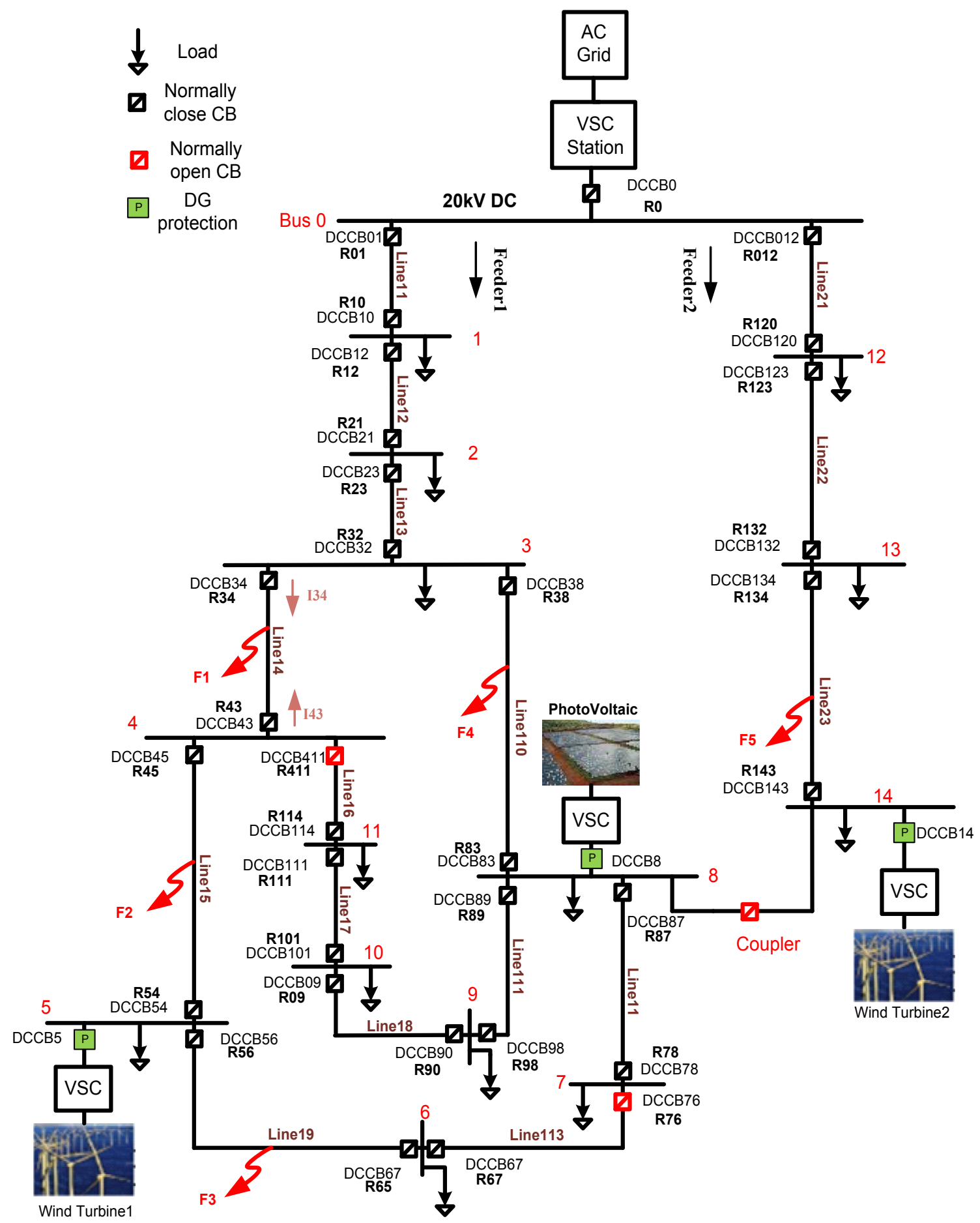

Fig. 11. Single-line diagram of the study distribution system. 

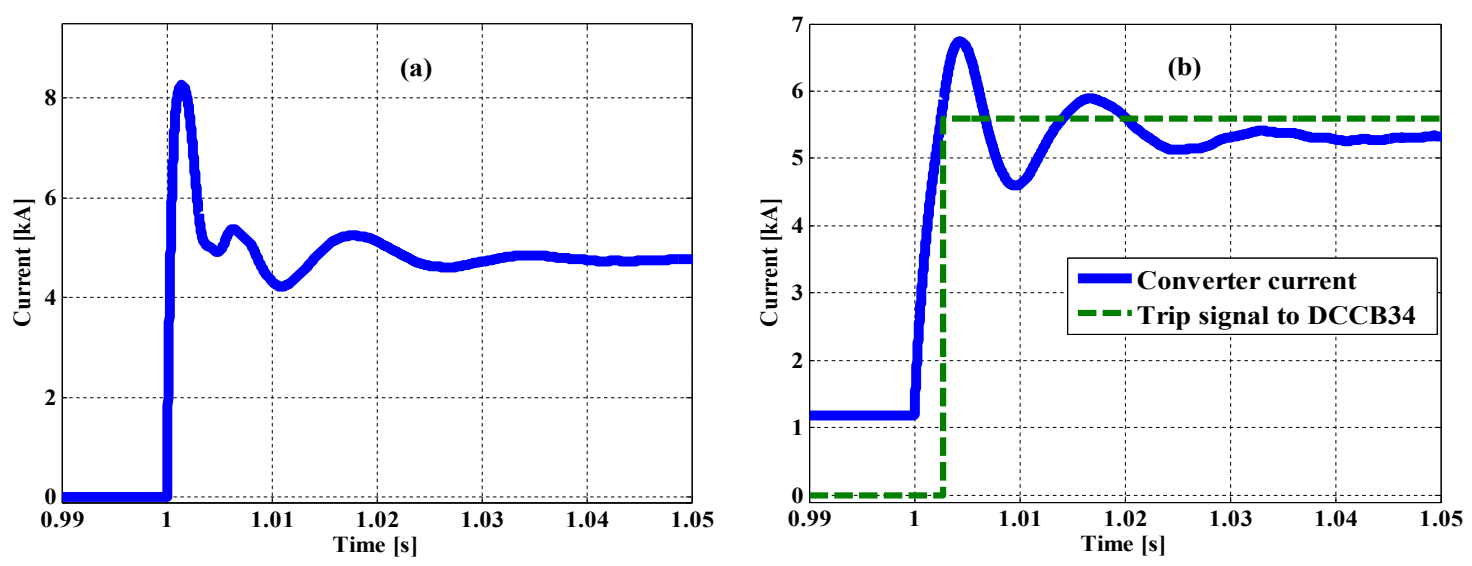

Fig. 12. Waveforms of (a) differential current seen by R34, and (b) converter current and scaled trip signal to DCCB34.

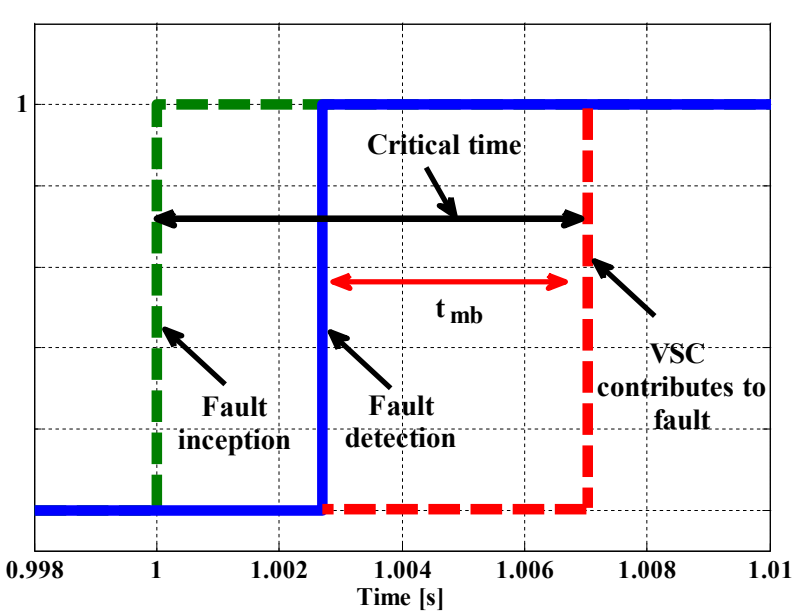

Fig. 13. Time delay in fault detection and sending the trip signal.

The study system is a bipolar DC network which includes two main DC feeders connected through a normally open coupler circuit breaker. Therefore, the loads can be supplied by radial or mesh lines depending on the status of DCCB411, DCCB76, and the coupler; these breakers normally are open. The two-level $\pm 10-\mathrm{kV}$ VSCs are used to interface the AC network and RESs to the DC network. Hall-Effect CTs installed at both sides of the DC lines determine the borders of the protection zones. The data transfer amongst the DC buses is made possible via dedicated communication links.

Each DG is individually protected so that it will be disconnected form the network if a fault strikes the grid for a certain period of time. Fig. 11 shows the location of DCCBs and relays. The loads are protected by fuses and the busbar protection scheme is not shown in the figure. Other parameters of the study system are reported in Tables 2 and 3, Appendix.

\subsection{Study cases}

In the following paragraphs, several fault scenarios are simulated to demonstrate the efficacy of the proposed method, considering communication delay. In all study cases, it is assumed that the fault is initiated at $\mathrm{t}=1.0 \mathrm{~s}$.

\subsubsection{Study case 1}

The objective of this case is to evaluate the performance of the proposed strategy when the communication medium operates

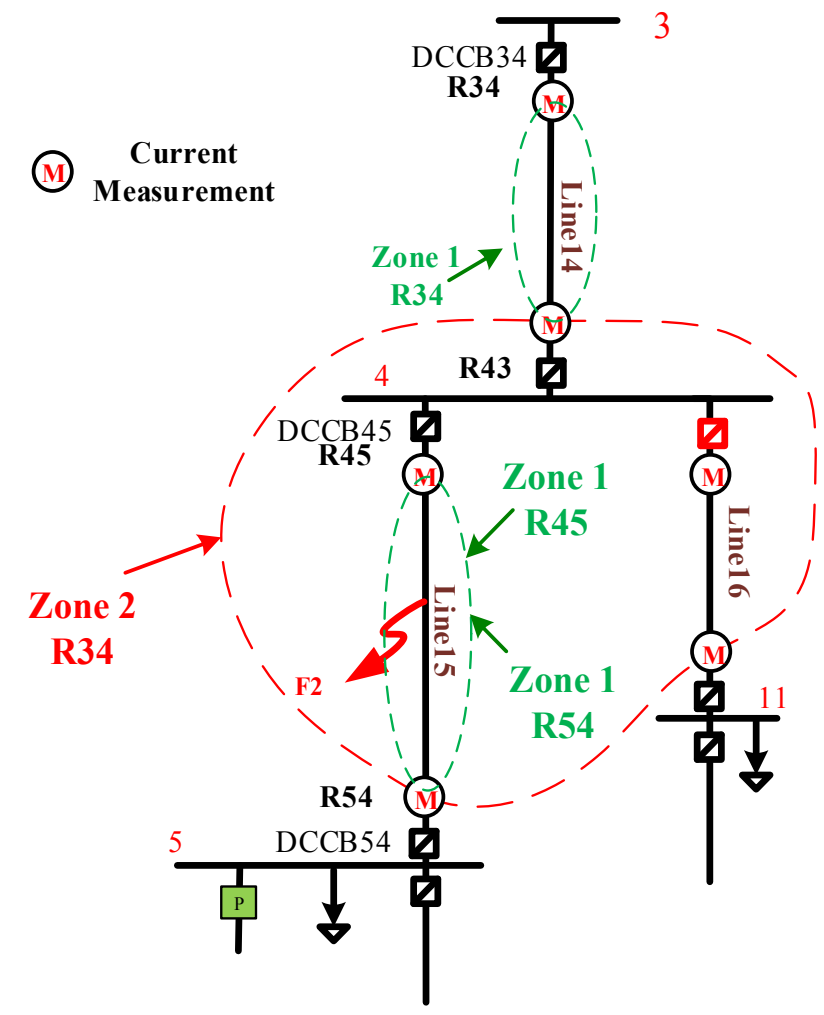

Fig. 14. The protection zones of R34, R45, and R54.

correctly. In this case, a PP fault impacts location F1 of the study system. Thus, the protection algorithm of relay R34 detects the fault in its first zone and sends a signal to the OPAL-RT, via Ethernet ports, to trip its corresponding DCCB. Fig. 12(a) shows that, subsequent to the PP fault occurrence, the differential current seen by R34 increases rapidly. The fault current of the VSC station is shown in Fig. 12(b); the figure indicates that the trip signal is generated by R34 during the capacitor discharging current (before the main converter can contribute to the fault); hence, the proposed protection is fast enough to prevent any damage to the VSC station.

The timing for the fault detection and VSC contribution is shown in Fig. 13. The figure indicates that relay R34 detects the fault in its main zone and sends the trip signal to DCCB34 almost $2.6 \mathrm{~ms}$ after the fault inception. This delay is due to the communication and processing times. Although the delays caused by 

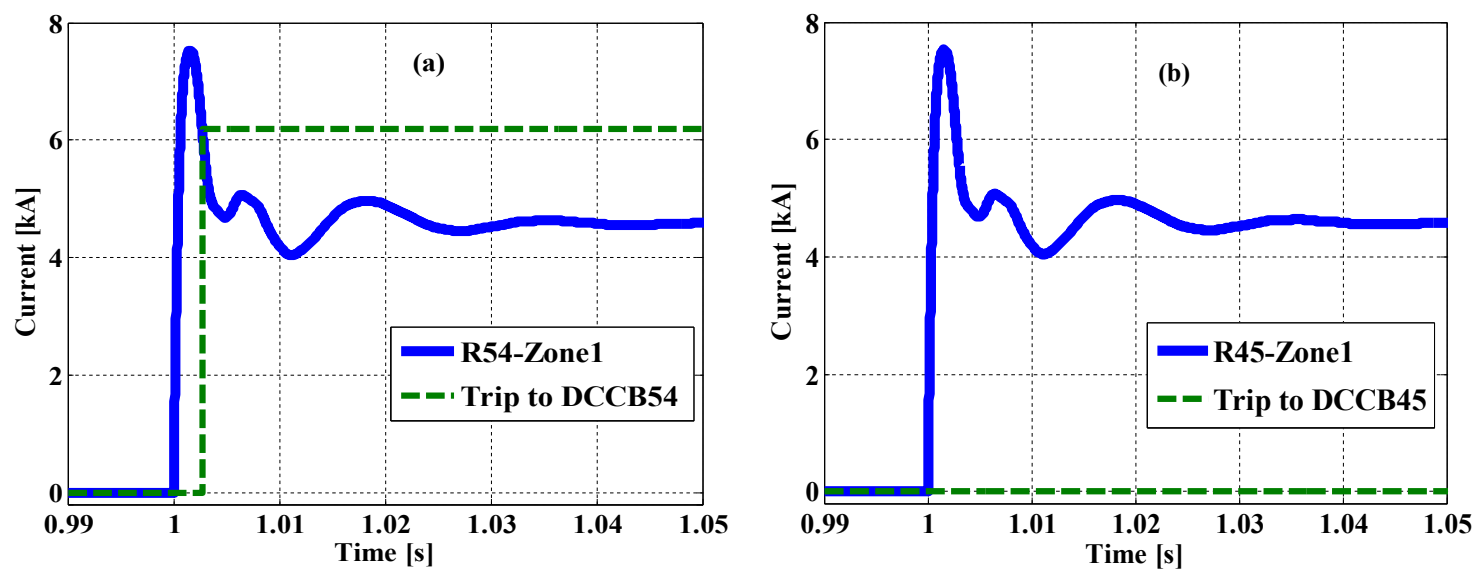

Fig. 15. The measured differential current and issued trip signals by (a) R54 (Zone 1), and (b) R45 (Zone 1).

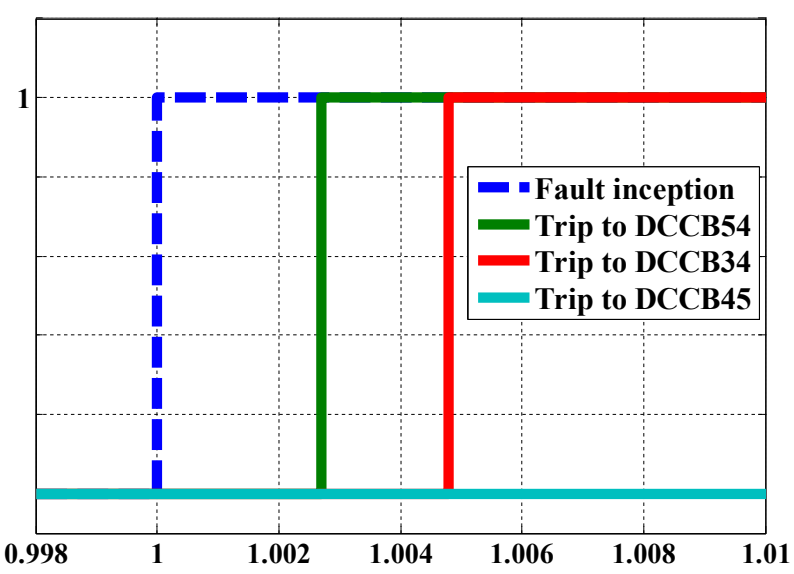

Fig. 16. Trip signals of the main and backup protection for a PP fault at F2.

measurement and interface devices are not considered in this study, it has been verified that such delays are less than $10 \mu \mathrm{s}$ assuming Hall-Effect CTs and appropriate microprocessor-based relays are used [33].

As shown in Fig. 13, $t_{\text {det }}$ is around $2.6 \mathrm{~ms}( \pm 0.3 \mathrm{~ms}$ in different test cases). Moreover, simulation results show that the critical time of the main converter of the study system for the solid PP fault is around $7.2 \mathrm{~ms}$. Therefore, once the fault is detected, it should be cleared in less than $4.6 \mathrm{~ms}( \pm 0.3 \mathrm{~ms})$, which is the maximum time that fault current, must be interrupted by the main or the backup protection. Therefore, the maximum value of TMD is calculated as [36]:

$T M D=t_{m b} / 2 \approx 2.3 m s$

On the other hand, since DCCBs are assumed to be of solid-state type, their operating time is less than $1 \mathrm{~ms}$ [39]. Therefore, considering some microseconds delay for measurement devices and A/D conversion and also a marginal time, the time delay between the main and backup differential zones is set on $2.0 \mathrm{~ms}$ in this study.

\subsubsection{Study case 2}

Let us now assume that a PP fault takes place at location F2, and Relay R45 fails to detect and isolate the fault. Thus, due to the R45 failure, the fault is detected by the backup protection, that is, Zone 2 of R34. Fig. 14 illustrates the protection zones of R45, R54, and R34.

Fig. 15(a) shows that Zone 1 of R54 detects the fault and sends the trip signal to DCCB54 in about $2.7 \mathrm{~ms}$. However, as shown in Fig. 15(b), Zone 1 of R45 fails to issue the trip signal. Hence, Zone 2 of R34 operates after a predefined time delay (i.e., TMD $=2 \mathrm{~ms}$ ). The main and the backup trip signals are shown in Fig. 16; the figure indicates that the backup trip is sent to DCCB34 in about $4.8 \mathrm{~ms}$ from the fault initiation.
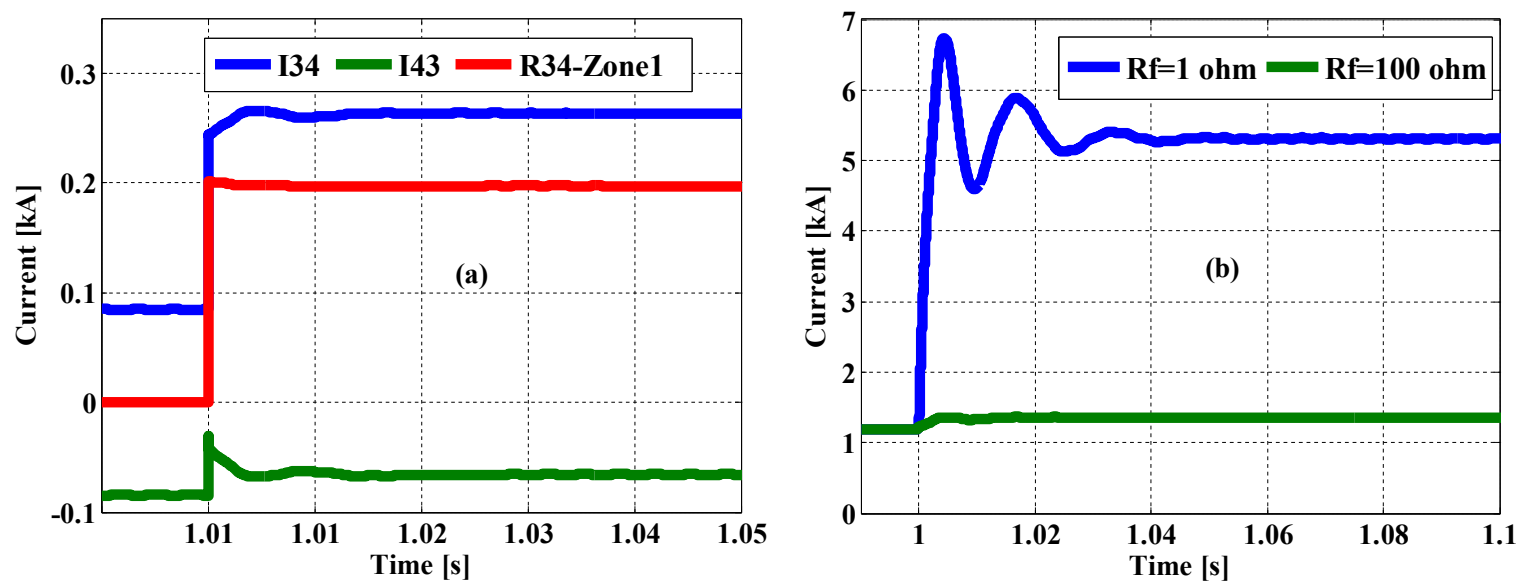

Fig. 17. Waveforms of (a) the fault and differential currents of the faulty line, and (b) the converter current for two fault resistance. 


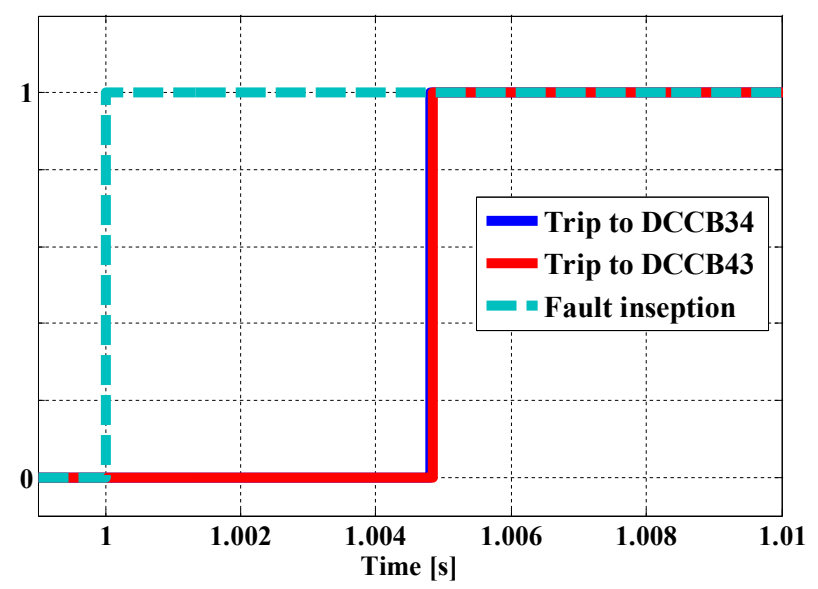

Fig. 18. Trip signals to the DCCBs.

\subsubsection{Study case 3}

To verify the effectiveness of the proposed method for highimpedance faults, it is assumed that a $100-\Omega$ PP fault strikes the study system at location F1. Fig. 17(a) shows the currents measured at both sides of the faulty line as well as the differential current of Zone 1 of R34. The effect of the fault resistance on the fault current measured at the VSC station is also shown Fig. 17(b). As indicated in
Fig. 18, relays R34 detects the fault and issues the trip signal in about $4.7 \mathrm{~ms}$ from the fault initiation. It is evident that, due to the impact of the fault resistant on the rising rate of the DC fault current, the relay operating time is relatively higher in this case; however, as mentioned in Section 2, it is still fast enough to ensure operation of the protection system before the critical time of VSC station.

\subsubsection{Study case 4}

As discussed in Section 3.2.2, the $\mathrm{O} / \mathrm{C}$ directional relays will provide backup protection if the communication network fails. Fig. 19 shows the protection zones of backup O/C directional units which have been selected based on the position of normally open DCCBs. Considering the selected backup protection zones, relays R01, R32, R34, R38, R012, and R134 are equipped with O/C directional units. It is, however, noted that $\mathrm{R} 32$ is equipped with the $\mathrm{O} / \mathrm{C}$ directional unit only to prevent undesired power interruption, in case of R34, R38 (or their associated DCCBs) failure. The remaining relays are disabled until the communication network is restored.

As illustrated in Fig. 19, the maximum operating time of the most upstream relay, i.e., R0, is 3TM. As explained in Section 3.2.2, the TM should be selected based on the critical time of the main VSC as well as constraints for coordination with other relays. Therefore, since the critical time of the main VSC is $7.2 \mathrm{~ms}$, the maximum value of TM turns out to be $2.4 \mathrm{~ms}$. Further, the operating time of the main protection of each zone can be expressed as:

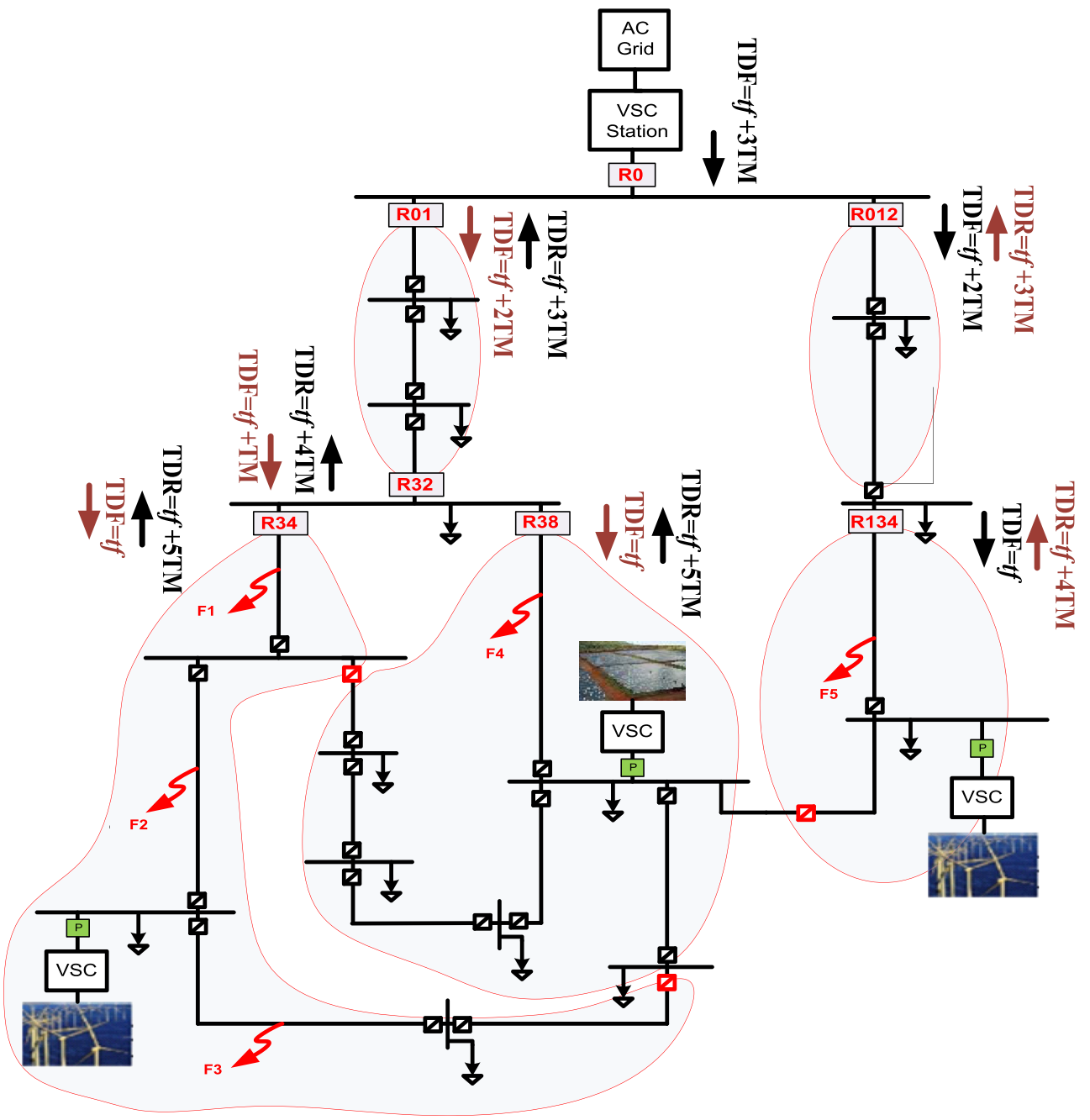

Fig. 19. Backup protection zones (in the case of communication network failure). 
Table 1

Operating times of the main and backup protection for selected fault scenarios.

\begin{tabular}{|c|c|c|c|c|c|c|c|c|}
\hline \multirow{2}{*}{$\begin{array}{l}\text { Fault } \\
\text { type }\end{array}$} & \multirow{2}{*}{$\begin{array}{l}\text { Fault } \\
\text { location }\end{array}$} & \multirow{2}{*}{$\begin{array}{l}\text { Fault } \\
\text { resistance }(\Omega)\end{array}$} & \multicolumn{3}{|c|}{ Main protection } & \multicolumn{3}{|c|}{ Backup protection } \\
\hline & & & Relay & Zone & Trip signal at $(m s)$ & Relay & Zone & Trip signal at $(m s)$ \\
\hline \multirow[t]{6}{*}{ PP } & F1 & 1.00 & R34 & Zone 1 & 2.6 & $\mathrm{R} 23$ & Zone 2 & 4.8 \\
\hline & $\mathrm{F} 1$ & 100 & R34 & Zone 1 & 4.8 & $\mathrm{R} 23$ & Zone 2 & 6.7 \\
\hline & F2 & 1.00 & R45 & Zone 1 & 2.4 & R34 & Zone 2 & 4.6 \\
\hline & F2 & 100 & $\mathrm{R} 45$ & Zone 1 & 4.4 & R34 & Zone 2 & 6.5 \\
\hline & F3 & 1.00 & R56 & Zone 1 & 2.5 & R45 & Zone 2 & 4.7 \\
\hline & F4 & 1.00 & $\mathrm{R} 38$ & Zone 1 & 2.5 & $\mathrm{R} 23$ & Zone 2 & 4.6 \\
\hline \multirow[t]{6}{*}{ PG } & $\mathrm{F} 1$ & 1.00 & R34 & Zone 1 & 2.3 & $\mathrm{R} 23$ & Zone 2 & 4.5 \\
\hline & F2 & 1.00 & $\mathrm{R} 45$ & Zone 1 & 2.7 & R34 & Zone 2 & 4.8 \\
\hline & F3 & 1.00 & R56 & Zone 1 & 2.6 & R45 & Zone 2 & 4.7 \\
\hline & F3 & 100 & R56 & Zone 1 & 4.5 & R45 & Zone 2 & 6.6 \\
\hline & F4 & 1.00 & R38 & Zone 1 & 2.5 & $\mathrm{R} 23$ & Zone 2 & 4.6 \\
\hline & F4 & 100 & R38 & Zone 1 & 3.7 & $\mathrm{R} 23$ & Zone 2 & 6.0 \\
\hline \multicolumn{9}{|c|}{ Communication Failure } \\
\hline $\mathrm{PP}$ & F1 & 1.00 & R34 & - & Inst. & R32 & - & 2 \\
\hline PP & F2 & 1.00 & R34 & - & Inst. & R32 & - & 2 \\
\hline PG & F5 & 1.00 & $\mathrm{R} 134$ & - & Inst. & R012 & - & 2.4 \\
\hline
\end{tabular}

$t_{o p}=t_{C B}+t_{d e t}+t_{C T}$

where $t_{C T}$ signifies the delay associated with current transducers and their corresponding A/D converters (several microseconds); $t_{d e t}$ is the fault detection time of the relay algorithm (in the range of several microseconds); and $t_{C B}$ denotes the operating time of DCCBs. Therefore, the main protection of each zone will operate in about $1 \mathrm{~ms}$, and considering an additional time margin, TM is set on $2 \mathrm{~ms}$ in this study. This TM is used to coordinate the selected relays which operate when the communication system fails.

To further demonstrate the effectiveness of the proposed protection strategy, various fault scenarios have also been tested using the real-time setup. However, the results of a selected number of test cases have been reported in Table 1 .

\section{Conclusions}

This paper proposes a multi-zone differential-based protection strategy for DC distribution networks. Given the special behaviour of DC fault currents that adversely affect the coordination of $\mathrm{O} / \mathrm{C}$ based relays, it has been proven that the proposed method provides a selective protection with a fast and simple algorithm. Therefore, by providing the higher selectivity, the proposed scheme minimizes the interrupted zones and improves the reliability of DC distribution systems. Moreover, the proposed protection scheme was also complemented with a backup protection to handle communication network failures.

Compared to the conventional differential-based methods, our proposed protection scheme enhances the reliability of the protection by introducing an additional differential backup for the neighbouring relays, and providing a backup protection which will manage communication failures. In addition, the salient feature of the proposed method is that its effectiveness is independent of the fault current level, fault impedance, operational mode of the network, as well as the type, size, location, and status of DGs.

Furthermore, a relay coordination method, for both the communication-based and backup units, has been presented in the paper where the operation of the main and backup zones as well as series relays are coordinated considering the critical time of the VSC stations, delay on communication and operating time of DCCBs.

Several fault cases were studied and analysed using a real-time HIL test setup to demonstrate the effectiveness of the proposed method. The HIL results confirmed that the proposed method has a high accuracy and is robust for the various fault types. It is also seen that, considering around $2.6 \mathrm{~ms}$ for the communication delay, both main and backup protections can detect the simulated faults within an acceptable time frame. Therefore, the proposed method is fast enough to ensure the safety of the VSC stations as well as network components.

\section{Acknowledgements}

This work was supported in part by the Spanish Ministry of Economy and Competitiveness under Project ENE2013-48428-C22-R. The work of M. Monadi was supported by the Ministry of Science, Research, and Technology, Iran. Any opinions, findings, and conclusions or recommendations expressed in this material are those of the authors and do not necessarily reflect those of the host institutions or founders.

\section{Appendix}

Table 2

Network cables, equivalent $\pi$, model parameters

\begin{tabular}{|c|c|c|c|c|c|c|c|c|c|}
\hline Line & $\mathrm{L}[\mathrm{kM}]$ & $\mathrm{R}[\Omega]$ & $\mathrm{X}[\Omega]$ & $\mathrm{C}[\mu \mathrm{F}]$ & Line & $\mathrm{L}[\mathrm{kM}]$ & $\mathrm{R}[\Omega]$ & $\mathrm{X}[\Omega]$ & $\mathrm{C}[\mu \mathrm{F}]$ \\
\hline Line11 & 2.82 & 0.355 & 0.319 & 0.448 & Line19 & 1.54 & 0.416 & 0.194 & 8.451 \\
\hline Line12 & 1.2 & 0.164 & 0.151 & 0.753 & Line 110 & 1.3 & 0.164 & 0.149 & 8.549 \\
\hline Line13 & 4.42 & 0.557 & 0.50 & 2.921 & Line111 & 0.32 & 0.086 & 0.132 & 1.398 \\
\hline Line14 & 0.61 & 0.119 & 0.073 & 3.953 & Line112 & 1.67 & 0.325 & 0.654 & 9.352 \\
\hline Line15 & 0.56 & 0.151 & 0.072 & 2.554 & Line113 & 0.24 & 0.047 & 0.099 & 0.902 \\
\hline Line16 & 0.49 & 0.132 & 0.066 & 2.430 & Line21 & 1.41 & 0.187 & 0.159 & 0.224 \\
\hline Line17 & 0.33 & 0.089 & 0.044 & 1.505 & Line22 & 4.89 & 1.320 & 1.750 & 0.796 \\
\hline Line18 & 0.77 & 0.208 & 0.102 & 3.721 & Line23 & 2.99 & 0.460 & 0.364 & 14.30 \\
\hline
\end{tabular}


Table 3

DC network parameters.

\begin{tabular}{|c|c|c|c|c|c|}
\hline Bus No. & $\begin{array}{l}\text { Installed power } \\
\text { generation }(\mathrm{kW})\end{array}$ & Load (kW) & Bus No. & $\begin{array}{l}\text { Installed power } \\
\text { generation }(\mathrm{kW})\end{array}$ & Load $(\mathrm{kW})$ \\
\hline Bus1 & - & 19,992 & Bus8 & 1000 & 586 \\
\hline Bus2 & - & 850 & Bus9 & - & 654 \\
\hline Bus3 & - & 501 & Bus10 & - & 543 \\
\hline Bus4 & - & 431 & Bus11 & - & 329 \\
\hline Bus5 & 1200 & 727 & Bus12 & - & 20,010 \\
\hline Bus6 & - & 548 & Bus13 & 1200 & 34 \\
\hline Bus7 & - & 76.5 & Bus14 & - & 9270 \\
\hline
\end{tabular}

\section{References}

[1] Yang Y, Zhang S, Xiao Y. Optimal design of distributed energy resource systems coupled with energy distribution networks. Energy 2015;85:433-48.

[2] Cai B, Liu Y, Ma Y, Huang L, Liu Z. A framework for the reliability evaluation of grid-connected photovoltaic systems in the presence of intermittent faults. Energy 2015;93:1308-20. Part 2.

[3] Dargahi V, Sadigh AK, Pahlavani MRA, Shoulaie A. DC (direct current) voltage source reduction in stacked multicell converter based energy systems. Energy 2012;46:649-63.

[4] De-Prada-Gil M, Díaz-González F, Gomis-Bellmunt O, Sumper A. DFIG-based offshore wind power plant connected to a single VSC-HVDC operated at variable frequency: energy yield assessment. Energy 2015;86:311-22.

[5] Sechilariu M, Wang BC, Locment F, Jouglet A. DC microgrid power flow optimization by multi-layer supervision control. Design and experimental validation. Energy Convers Manag 2014;82:1-10.

[6] Humpert C. Long distance transmission systems for the future electricity supply - analysis of possibilities and restrictions. Energy 2012;48:278-83.

[7] Deng LH, Wang ZX, Duan JM. Protection scheme for DC microgrid distribution system. In: Advanced materials research; 2013. p. 1661-5.

[8] Asad R, Kazemi A. A novel distributed optimal power sharing method for radial dc microgrids with different distributed energy sources. Energy 2014;72:291-9.

[9] Majumder R. Aggregation of microgrids with DC system. Electr Power Syst Res 2014; $108: 134-43$.

[10] Fletcher SDA, Norman PJ, Galloway SJ, Crolla P, Burt GM. Optimizing the roles of unit and non-unit protection methods within DC microgrids. IEEE Trans Smart Grid Dec 2012;3:2079-87.

[11] Seixas M, Melício R, Mendes VMF. Offshore wind turbine simulation: multibody drive train. Back-to-back NPC (neutral point clamped) converters. Fractional-order control. Energy 2014;69:357-69.

[12] Yang J, Fletcher JE, O'Reilly J. Short-circuit and ground fault analyses and location in VSC-based DC network cables. Industrial Electron IEEE Trans 2012:59:3827-37.

[13] Zeng B, Wen J, Shi J, Zhang J, Zhang Y. A multi-level approach to active distribution system planning for efficient renewable energy harvesting in deregulated environment. Energy 2016;96:614-24.

[14] Ahmadigorji M, Amjady N. A multiyear DG-incorporated framework for expansion planning of distribution networks using binary chaotic shark smel optimization algorithm. Energy 2016;102:199-215.

[15] Monadi M, Amin Zamani M, Ignacio Candela J, Luna A, Rodriguez P. Protection of AC and DC distribution systems Embedding distributed energy resources: a comparative review and analysis. Renew Sustain Energy Rev 2015;51:1578-93.

[16] Salomonsson D, Soder L, Sannino A. Protection of low-voltage DC microgrids Power Deliv IEEE Trans 2009;24:1045-53.

[17] Baran ME, Mahajan NR. Overcurrent protection on voltage-source-converter-based multiterminal DC distribution systems. Power Deliv IEEE Trans 2007;22:406-12.

[18] Fletcher S, Norman P, Galloway S, Burt G. Fault detection and location in DC systems from initial di/dt measurement. In: Euro Tech Con Conference; 2012 https://pure.strath.ac.uk/portal/files/26225420/Euro_TechCon_di_dt_fault_ detection_Steven_Fletcher.pdf.

[19] Farhadi M, Mohammed OA. Event-based protection scheme for a multiterminal hybrid DC power system. IEEE Trans Smart Grid Jul 2015;6:1658-69.

[20] Leterme W, Beerten J, Van Hertem D. Non-unit protection of HVDC grids with inductive dc cable termination. IEEE Trans Power Deliv 2015;31(2). 820-828.

[21] Christopher E, Sumner M, Thomas DWP, Xiaohui W, de Wildt F. Fault location in a zonal DC marine power system using active impedance estimation. Industry Appl IEEE Trans 2013;49:860-5.

[22] Liu Z, Liu Y, Zhang D, Cai B, Zheng C. Fault diagnosis for a solar assisted heat pump system under incomplete data and expert knowledge. Energy 2015;87: 41-8.

[23] Chanda NK, Fu Y. ANN-based fault classification and location in MVDC shipboard power systems. In: North American power symposium (NAPS), 2011; 2011. p. $1-7$.
[24] Li WL, Monti A, Ponci F. Fault detection and classification in medium voltage DC shipboard power systems with wavelets and artificial neural networks. IEEE Trans Instrum Meas Nov 2014;63:2651-65.

[25] De Kerf K, Srivastava K, Reza M, Bekaert D, Cole S, Van Hertem D, et al. Wavelet-based protection strategy for DC faults in multi-terminal VSC HVDC systems. Generation, Transm Distribution, IET 2011;5:496-503.

[26] Gab-Su S, Hyunsu B, Bo-Hyung C, Kyu-Chan L. Arc protection scheme for DC distribution systems with photovoltaic generation. In: Renewable energy Research and applications (ICRERA), 2012 international conference on; 2012. p. $1-5$.

[27] Azizi S, Afsharnia S, Sanaye-Pasand M. Fault location on multi-terminal DC systems using synchronized current measurements. Int J Electr Power \& Energy Syst 2014;63:779-86.

[28] Silvestre S, Kichou S, Chouder A, Nofuentes G, Karatepe E. Analysis of current and voltage indicators in grid connected PV (photovoltaic) systems working in faulty and partial shading conditions. Energy 2015;86:42-50.

[29] Park JD, Candelaria J, Ma LY, Dunn K. Dc ring-bus microgrid fault protection and identification of fault location. IEEE Trans Power Deliv Oct 2013;28: 2574-84.

[30] Personal E, Guerrero JI, Garcia A, Peña M, Leon C. Key performance indicators: a useful tool to assess Smart Grid goals. Energy 2014;76:976-88.

[31] Arasteh H, Sepasian MS, Vahidinasab V. An aggregated model for coordinated planning and reconfiguration of electric distribution networks. Energy 2016;94:786-98.

[32] Nikolaidis VC, Papanikolaou E, Safigianni AS. A communication-assisted overcurrent protection scheme for radial distribution systems with distributed generation. Smart Grid, IEEE Trans 2016;7:114-23.

[33] Fletcher SDA, Norman PJ, Fong K, Galloway SJ, Burt GM. High-speed differential protection for smart DC distribution systems. Smart Grid, IEEE Trans Sep 2014;5:2610-7.

[34] Hajian M, Zhang L, Jovcic D. DC transmission grid with low-speed protection using mechanical DC circuit breakers. IEEE Trans Power Deliv 2015;30: 1383-91.

[35] Abu-Elanien AEB, Elserougi AA, Abdel-Khalik AS, Massoud AM, Ahmed S, A differential protection technique for multi-terminal HVDC. Electr Power Syst Res 2016;130:78-88.

[36] Yang J, Fletcher JE, O'Reilly J. Multiterminal DC wind farm collection grid internal fault analysis and protection design. Power Deliv IEEE Trans 2010;25: 2308-18.

[37] Tang L, Ooi B-T. Locating and isolating DC faults in multi-terminal DC systems. Power Deliv IEEE Trans 2007;22:1877-84.

[38] Bucher MK, Franck CM. Fault current interruption in multiterminal HVDC networks. Power Deliv IEEE Trans 2016:31:87-95.

[39] Franck CM. HVDC circuit breakers: a review identifying future research needs. Power Deliv IEEE Trans 2011;26:998-1007.

[40] Anderson PM. Power system protection, vol. 1307. New York: McGraw-Hill; 1999.

[41] Fletcher S, Norman P, Galloway S, Burt G. Determination of protection system requirements for DC unmanned aerial vehicle electrical power networks for enhanced capability and survivability. IET Electr Syst Transp 2011;1:137-47.

[42] Zamani MA, Yazdani A, Sidhu TS. A communication-assisted protection strategy for inverter-based medium-voltage microgrids. Smart Grid, IEEE Trans 2012:3:2088-99.

[43] IEC 61850-8-1. In: IEC, editor. Communication networks and systems in substations - Part 8-1: specific communication service mapping (SCSM) mappings to MMS (ISO 9506-1 and ISO 9506-2) and to ISO/IEC 8802-3; 2004.

[44] Sortomme E, Venkata S, Mitra J. Microgrid protection using communicationassisted digital relays. Power Deliv, IEEE Trans 2010;25:2789-96.

[45] Rudion K, Orths A, Styczynski Z, Strunz K. Design of benchmark of medium voltage distribution network for investigation of DG integration. In: Power energy society general meeting, 2006. IEEE; 2006. 6 pp. 